Research Paper

\title{
ATM Expression Is Elevated in Established Radiation-Resistant Breast Cancer Cells and Improves DNA Repair Efficiency
}

\author{
Lei Bian ${ }^{*}$, Yiling Meng1 ${ }^{*}$, Meichao Zhang1 ${ }^{*}$, Zhuying Guo ${ }^{2}$, Furao Liu1, Weiwen Zhang1, Xue Ke1, Yuxuan \\ $\mathrm{Su}^{1}$, Meng Wang1, Yuan $\mathrm{Yao}^{1 \bowtie}$, Lizhong $\mathrm{Wu}^{3 凶}$, Dong Li ${ }^{1 凶}$ \\ 1. Department of Radiation Oncology, Shanghai Ninth People's Hospital, Shanghai Jiaotong University School of Medicine, Shanghai, China \\ 2. Department of Clinical Laboratory, Shanghai Ninth People's Hospital, Shanghai Jiaotong University School of Medicine, Shanghai, China \\ 3. Department of Radiology, Shanghai Ninth People's Hospital, Shanghai Jiaotong University School of Medicine, Shanghai, China \\ *These authors contributed equally to this work.
}

$\triangle$ Corresponding authors: Dong Li, Department of Radiation Oncology, Shanghai Ninth People's Hospital, Shanghai Jiaotong University School of Medicine, Shanghai, China; E-mail: lidong@shsmu.edu.cn. Lizhong Wu, Department of Radiology, Shanghai Ninth People's Hospital, Shanghai Jiaotong University School of Medicine, 280 Mohe Road, Shanghai, China, 201999. E-mail: woolley@shsmu.edu.cn. Yuan Yao, Department of Radiation Oncology, Shanghai Ninth People's Hospital, Shanghai Jiaotong University School of Medicine, Shanghai, China; E-mail: yaoyuan@shsmu.edu.cn.

(c) The author(s). This is an open access article distributed under the terms of the Creative Commons Attribution License (https://creativecommons.org/licenses/by/4.0/). See http://ivyspring.com/terms for full terms and conditions.

Received: 2019.10.16; Accepted: 2020.01.06; Published: 2020.02.04

\begin{abstract}
Repair of damaged DNA induced by radiation plays an important role in the development of radioresistance, which greatly restricts patients' benefit from radiotherapy. However, the relation between radioresistance development and DNA double-strand break repair pathways (mainly non-homologous end joining and homologous recombination) and how these pathways contribute to radioresistance are unclear. Here, we established a radioresistant breast cancer cell line by repeated ionizing radiation and studied the alteration in DNA repair capacity. Compared with parental sham-treated cells, radioresistant breast cancer cells present elevated radioresistance, enhanced malignancy, increased expression of Ataxia-telangiectasia mutated (ATM), and increased DNA damage repair efficiency, as reflected by accelerated $\mathrm{Y}-\mathrm{H} 2 \mathrm{AX}$ kinetic. These defects can be reversed by ATM inhibition or ATM knockdown, indicating a potential link between ATM, DNA repair pathway and radiosensitivity. We propose that cancer cells develop elevated radioresistance through enhanced DNA damage repair efficiency mediated by increased ATM expression. Our work might provide a new evidence supporting the potential of ATM as a potential target of cancer therapy.
\end{abstract}

Key words: breast cancer, radiation, DNA damage repair, ATM

\section{Introduction}

Radiation therapy is efficacious, cost-effective for many solid tumors. About $50 \%$ of cancer patients receive radiation therapy and $40 \%$ of cancer cure is believed to be related to radiation treatment [1, 2]. For example, along with chemotherapy, radiation therapy brings in a benefit of an absolute $10 \%$ reduction in the risk of disease-related death for breast cancer patients $[3,4]$. However, an adaptive response of cancer cells to radiation leads to the development of radiation resistance which ultimately leads to tumor recurrence and metastasis. As a result, the application of radiation therapy is still limited [5].

The anticancer efficacy of radiation is mainly due to its ionization effect. It produces oxygen free radicals and the latter causes a broad spectrum of DNA lesions [6]. Amongst lesions induced by ionizing radiation, double-strand breaks (DSBs) are the most lethal type, which could lead to cell apoptosis or necrosis [7]. To repair DSBs, two main DNA damage repair (DDR) pathways, homologous 
recombination (HR) and non-homologous end joining (NHEJ), are activated. These two main DSBs repair pathways play an indispensable role in initiating and processing of cancer. Patients harboring deficiencies affecting $\mathrm{HR}$ or NHEJ often present increased susceptibility towards cancer [8, 9]. Nonetheless, alterations in DSB repair pathways play an important role in the development of radioresistance [10].

$\mathrm{H} 2 \mathrm{AX}$ is a key component in DNA repair, which is rapidly phosphorylated on a serine four residues from the carboxyl terminus and forms $\gamma-\mathrm{H} 2 \mathrm{AX}$ at nascent DSB sites. During a short time after DSB formation, $\mathrm{\gamma}-\mathrm{H} 2 \mathrm{AX}$ accumulates around the breaks and forms foci that could be detected [11]. $\gamma-\mathrm{H} 2 \mathrm{AX}$ correlates well with DSBs formed. When DSBs are repaired, $y-\mathrm{H} 2 \mathrm{AX}$ disappears. Thus, the kinetics of $\mathrm{Y}-\mathrm{H} 2 \mathrm{AX}$ is often used to monitor DSBs repair efficiency [12]. NHEJ is the fast DSB repair pathway, which mainly contributes to the repair of DSBs in 2 hours after DSB formation [13]. Furthermore, NHEJ is believed to be the preferred pathway for the repair of IR-induced DSBs [14]. Some research indicated that it is HR repair efficiency, not NHEJ, that is significantly elevated in breast cancer cells compared with normal mammalian cells [15]. However, little is done to compare HR and NHEJ efficiency in relatively radio-resistant and radio-sensitive cells from the same origin, which might better reflect how the adaptation of radiation is developed in cancer cells.

Ataxia-telangiectasia mutated (ATM) encodes a serine/threonine kinase that belongs to the phosphatidyl inositol 3-kinase (PI3K) like protein kinases (PIKKs) family [16]. It functions as an important DSBs sensor [17]. In the HR pathway, many essential effectors are phosphorylated and activated by ATM. ATM also functions in the NHEJ pathway through phosphorylating kinase DNA-PKcs and nuclease Artemis [18]. Several studies showed that there was some associations between radiosensitivity and ATM expression levels [19-21]. Upregulation of ATM protein and its activation are reported to be correlated with enhanced radioresistance [22-24]. A combination of chemotherapy and ATM inhibitor is a promising new cancer treatment under trials nowadays [25].

In our present work, we established a relatively radio-resistant breast cancer cell line and found that $\mathrm{\gamma}-\mathrm{H} 2 \mathrm{AX}$ kinetic was significantly altered in the cells, indicating an elevated DNA damage repair response. Further experiments suggested that this might be mediated by ATM, a molecule vital for HR and NHEJ. Thus, the prediction of instinct sensitivity towards ionization therapy based on detecting DSB repair efficiency of cancer cells or monitoring ATM expression might be a promising new strategy for cancer treatment.

\section{Methods}

\section{Antibodies and reagents}

The following reagents were used: I-Scel enzyme (NEW ENGLAND Biolabs, \#R0694S). TIANgel Midi Purification Kit (TIANGEN, \#DP209-02), Hieff Trans $^{\mathrm{TM}}$ Liposomal Transfection Reagent (Yeasen, \#40802ES08), Apoptosis Analysis Kit (Yeasen, \#40301 ES50), CCK-8 kit (DOJINDO, \#CK04-3000T). Paclitaxel (\#HY-B0015), Doxorubicin (\#HY-15142A) and CP-466722(HY-11002) were purchased from MedChemExpress and dissolved in dimethyl sulfoxide (DMSO) for stocking solutions and stored according to manufacturer's instructions.

Antibodies used in western blotting and immunofluorescence are listed in Table. S1.

\section{Cell cultures and the establishment of radioresistant cell line}

Breast cancer cell line MDA-MB231 was purchased from ATCC and cultured following the protocol: cultured with the medium of DMEM medium (Gibco) supplemented with $10 \%$ fetal bovine serum (FBS) and maintained in a humidified atmosphere at $37^{\circ} \mathrm{C}$ with $5 \% \mathrm{CO}_{2}$.

For the establishment of the radioresistant cell line, parental MDA-MB-231 cells were divided into two groups, MDA-MB-231-PB and MDA-MB-231-PR. $1 \times 10^{6}$ cells were seeded in $10 \mathrm{~cm}$ culture disks on day 1. On day 3 or 4 , when cells attained $60-70 \%$ confluency, MDA-MB-231-PR cells received 3Gray (Gy) of X-ray (Elekta) irradiation at a dose rate of 1.43Gy per minute. Cells were trypsinized, counted and passed into new dishes when cell confluency reached approximately $90 \%$. Medium was changed every other day. The irradiation was performed 20 times for a total dose of 60Gy over 4 months. MDA-MB-231-PB cells were treated under the same condition without the irradiation.

\section{Clonogenic survival and linear-quadratic-model}

Proper numbers of cells were plated in 6-wells cell culture plates in triplicate and allowed 24 hours for attachment. After 24 hours, cells were irradiated with $0-6 \mathrm{~Gy}$ of X-ray at a dose rate of $1.43 \mathrm{~Gy}$ per minute and then incubated 10 to 14 days for colony formation. Colonies were fixed with $4 \%$ paraformaldehyde and stained with crystal violet. Colonies of $>50$ cells were counted as clonogenic survivors. Surviving fraction (SF) after a certain dose was calculated. $\mathrm{SF}(\mathrm{d})=$ [number of clonogenic survivor (d)] / [number of clonogenic survivors (0)] while $\mathrm{d}$ indicates radiation dose and 0 means when dose is 0 . Linear-quadratic -model is described below: 


$$
S=e^{-d(\alpha+\beta d)}
$$

Here, e represents the natural logarithm, with $\mathrm{S}$ is the surviving fraction after a certain dose of ionizing radiation.

\section{Western blotting}

Western blotting was performed according to a standard protocol. Briefly, cells were lysed in lysis buffer [26] and were analyzed by immunoblotting after SDS-PAGE. Proteins were visualized by ECL according to the manufacturer's instructions (Millipore).

\section{Immunofluorescence staining}

Cells were seeded on coverslips placed in 12-well plates. 24 hours later, cells were irradiated as indicated. Cells were then fixed, permeabilized and blocked at the indicated time. Anti-Phosphor-Histone H2A.X(Ser139) and Donkey anti-mouse IgG Alexa Fluor conjugate were used for staining. Dapi was used for nuclear staining. Pictures were taken using a Nikon A1 confocal microscope.

\section{Homologous recombination and Non-Homologous End Joining repair efficiency}

Plasmids for the detection of HR and NHEJ were generously provided by Dr. Vera Gorbunova (University of Rocherster, New York). Experiments were described previously [15]. In brief, the reporter substrate contains a GFP sequence with an inserted intron, which does not affect GFP expression. The intron is interrupted by an adenoviral exon, which is flanked by restriction sites of I-SceI for DSBs. Upon treatment with I-SceI, these restriction sites are recognized and digested, thus the adenoviral exon is cut out, DSBs are left which could be repaired by either HR or NHEJ. When the DSBs are repaired, the intron is ligated and the GFP expression is restored.

NHEJ-Incompatible (NHEJ-I), NHEJ-Compatible (NHEJ-C), and HR plasmids were linearized using ISceI enzyme. 1ug linearized plasmids and 1ug DsRed plasmid (used as transfection efficiency control) were co-transfected into cells. 72 hours later, cells were trypsinized and washed with cold phosphatebuffered saline (PBS), and subjected to flow cytometry analysis. The repair efficiency was calculated as the number of cells with green fluorescence/the number of cells with red fluorescence.

\section{siRNA knockdown}

Small interfering RNA (siRNA) transfection was carried out using Lipofectamine 3000 (Thermo Fisher Scientific). siRNA nucleotides against negative control (NC) and ATM were obtained from GenePharma. The sequence of siRNA oligonucleotides against ATM was
5'-GCU UGA GGC UGA UCC UUA UTT-3' (Si-1), and 5'-GCA AAG CCC UAG UAA CAU ATT-3'(Si-2).

\section{Flow cytometry}

Flow cytometry was done using FACS Canto ${ }^{\mathrm{TM}}$ flow cytometer. Cell cycle analysis was performed using the Cell Cycle and Apoptosis Analysis Kit (Yeasen, \#40301ES50) according to the manufacturer's instructions. Apoptosis analysis was performed using Annexin V-FITC/PI kit (Yeasen, \#40302ES20). Cells were subjected to radiation exposure or sham-treated. After the indicated time of incubation, cells were trypsinized and washed with PBS, then dyed with Annexin V-FITC/PI reagents before the flow cytometry analysis.

\section{Statistical analysis}

Results were expressed as mean \pm SD. Data were analyzed by using student's test. $P$ values $<0.05$ were considered significant.

\section{Results}

\section{Establishment of radioresistant breast cancer cell line}

Parental MDA-MB-231 cells were divided into two subsets. One received 20 times of fractioned irradiation with a total dose of 60Gy and designated as MDA-MB231-PR (MD-PR) cells. Another group was treated under the same condition but received no irradiation and named as MDA-MB231-PB (MD-PB) cells.

The morphology of MD-PR cells was obviously different from that of MD-PB cells under microscopy (Fig. 1A). MD-PR cells had a much more stretched and flatter appearance compared with the latter. We then examined whether the abilities of migration and invasion were changed in MD-PR cells. Results showed that MD-PR cells had increased migration and invasion capacities compared with MD-PB cells (Fig. S1A, B). Increased expression of mesenchymal markers (N-cadherin, Snail, Slug and beta-catenin) and decreased expression of epithelial marker E-cadherin in MD-PR were also detected (Fig. S1C). These results suggested that enhanced malignancy was induced in MD-PR cells [27].

The radiosensitivity of MD-PB cells and MD-PR cells was compared. Various parameters reflected that the MD-PR cell line had a higher survival rate compared with MD-PB cells (Fig. 1B, C, D). The surviving fraction at 2 Gy (SF2) is a commonly used parameter for measurement of radiosensitivity in vitro $[28,29]$. SF2 was significantly increased in MD-PR cells compared with MD-PB cells $(0.71 \pm 0.11$ vs $0.49 \pm 0.05, p=0.04$ ) (Fig. 1D) along with significant 
changes in SF4. Enhanced radioresistance in MD-PR cells was furtuher evidenced by apoptotic assays. Results of apoptotic assays showed that, after a large dose of irradiation, the proportion of pro-apoptotic (Annexin V-FITC positive) and apoptotic (Annexin VFITC positive, PI positive) cells was significantly reduced in MD-PR cells compared with MD-PB cells $(13.22 \pm 2.17$ vs $20.92 \pm 1.33, p=0.01$, Fig. $1 \mathrm{E})$. The above results showed that MB-PR cells were more radioresistant compared with MD-PB cells.

At the same time, we tested whether repeated irradiation could change the cell proliferation rate and cell cycle distribution. The results showed that there was no significant difference between MD-PR cells and MD-PB cells in these aspects (Fig. S1D, E).

\section{Altered $\mathrm{Y}-\mathrm{H} 2 \mathrm{AX}$ kinetic in radioresistant MD-PR cells}

To find the possible mechanism explaining reduced apoptosis ratio in MD-PR cell line after irradiation, DSB repair efficiency was evaluated in MD-PB and MD-PR cells by detecting Phosphor-
Histone H2A.X (Ser139) ( $\gamma-\mathrm{H} 2 \mathrm{AX})$, which was a widely adopted marker for the detection of DSBs [11].

Western blotting experiments evidenced that kinetics of $\gamma-\mathrm{H} 2 \mathrm{AX}$ varied between MD-PR and M-PB cells (Fig. 2A, B). $\gamma$-H2AX expression peaked at 15 minutes after irradiation and decreased to normal level at about 2-hour post-irradiation in MD-PR cells, while it attained a peak at 1-hour and decreased to normal level at 2-hour in MD-PB cells (Fig. 2B). The two patterns of $\gamma-\mathrm{H} 2 \mathrm{AX}$ kinetics suggested that DSBs were repaired faster in MD-PR cells. Consistent with results of western blotting, the number of $\mathrm{y}-\mathrm{H} 2 \mathrm{AX}$ foci per nuclei enumerated at 1-hour post-irradiation in MD-PR cells ( $30 \pm 7$ foci per nuclei) was significantly lower than that in MD-PB (44 \pm 11 foci per nuclei) (Fig. 2C, D).

These results suggested an enhanced DSB repair response in MD-PR cells, which may explain the increased survival fraction in this cell line after different dose of irradiation.
A

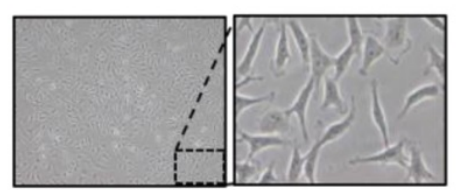

B

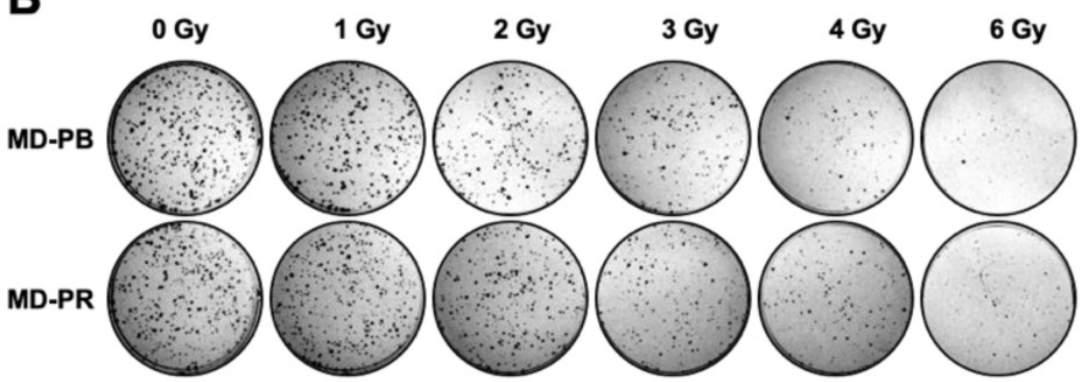

C

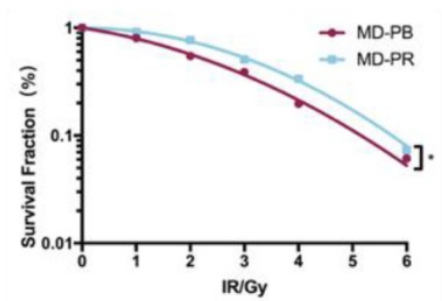

MD-PR

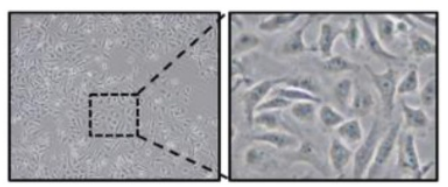

D

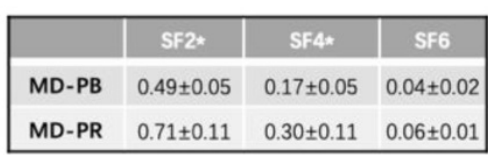

$\mathbf{E}$
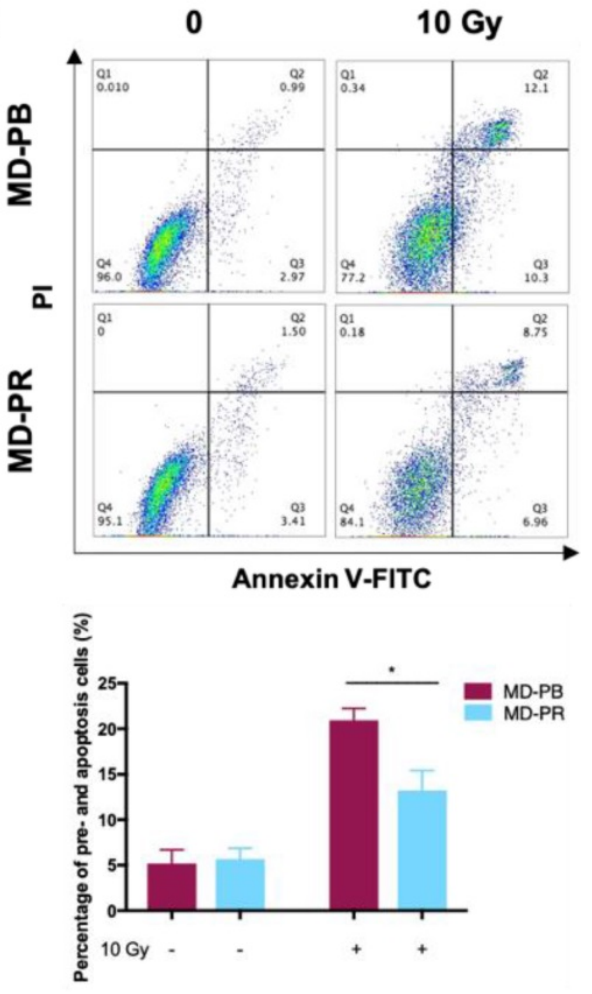

Figure 1. MD-PR cells present enhanced radioresistance compared with MD-PB. (A) Morphology changes of MD-PB and MD-PR under the microscope of $100 \mathrm{X}$ magnification, with representative cells zoomed in on the right. (B-D) MD-PB and MD-PR cells were seeded in a 6-well plate in triplicate. 24-hours later, cells were subjected to 0-6 Gy of X-ray radiation (Elekta, $1.43 \mathrm{~Gy} / \mathrm{min}$ ). After 10-14 days of incubation, formed clones were fixed, stained and counted. Surviving fraction was calculated and fitted into the linear-quadratic model as described in the Materials and Methods (C). A representative image of three independent experiments was showed (B). Surviving fraction at certain doses as indicated in (D). (E) MD-PB and MD-PR cells were exposed to 0 or 10 Gy of X-ray. 48 hours later, cells were collected, stained with PI and Annexin V-FITC dye and subjected to flow cytometry analysis. Annexin-V-positive cells (Q3) were counted as pre-apoptotic cells and PI-positive, Annexin-V-positive cells (Q2) were apoptotic. Percentage of apoptotic cells equals the sum of Q2 and Q3. Top panel: one representative result of apoptosis analysis. Bottom panel: the statistic results of 3 separate experiments. $(* p<0.05)$ 
A
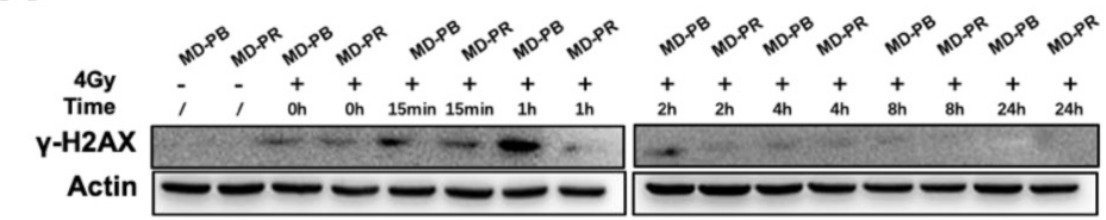

C
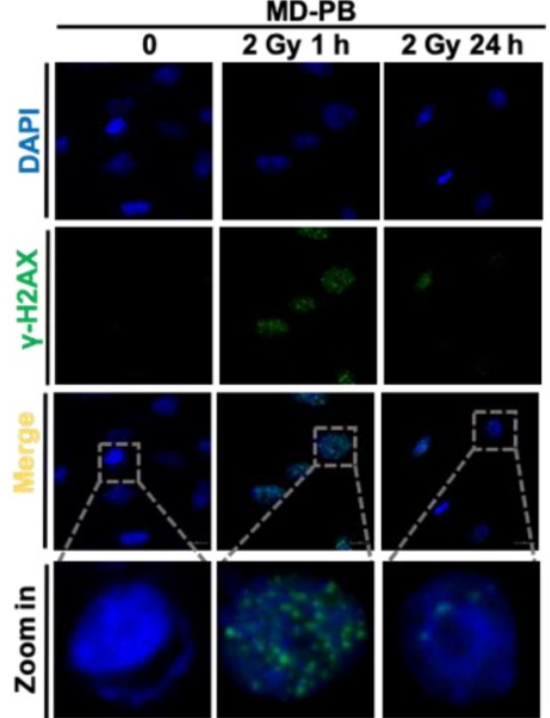
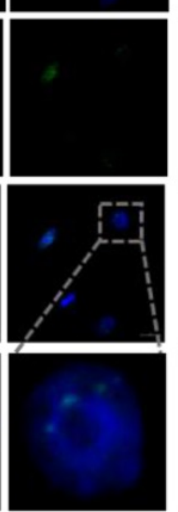

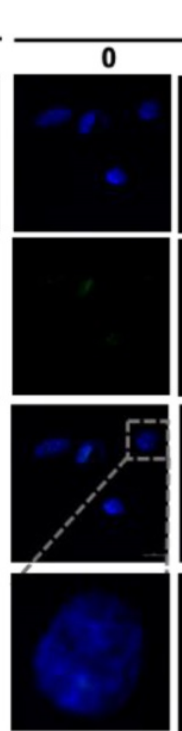

MD-PR

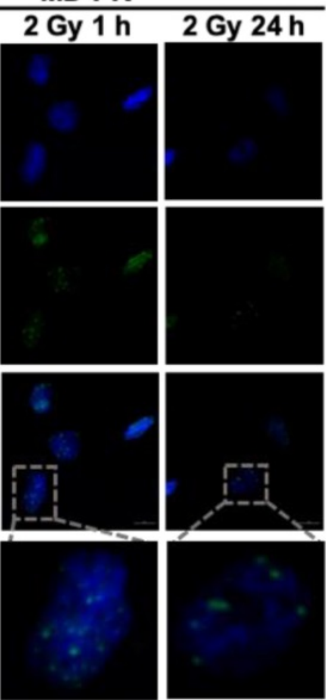

B

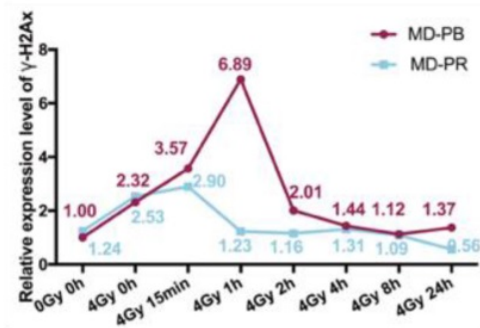

Figure 2. Altered Y-H2AX kinetic in MD-PR cells. (A) Cells were exposed to 0 or 4 Gy of irradiation and lysed at the indicated times for analysis of $Y-H 2 A X$ with Western-blotting. Actin was used as loading control. (B) Quantitative analysis of the blots. Plotted is the relative increase of $\mathrm{Y}-\mathrm{H} 2 \mathrm{AX}$ compared with MD-PB at $0 \mathrm{~Gy}$ as a function of post-irradiation incubation time, with statistics on the bottom. (C) Immunofluorescence of $\mathrm{Y}-\mathrm{H} 2 \mathrm{AX}$ in cells treated with or without $2 \mathrm{~Gy}$ of radiation. Samples were collected at indicated time. $\mathrm{Y}-\mathrm{H} 2 \mathrm{AX}$ was stained with green and nucleus with blue. Pictures were taken under 1600x magnification, with representative cells zoomed in at the bottom. Foci formed in nuclei was counted in at least 5 random fields and the statistical results were shown in $\mathrm{D}$. $(* * p<0.001)$

\section{ATM was up-regulated in radioresistant MD-PR cells}

Patterns of kinetic of $\mathrm{y}$-H2AX mainly differ within 2 hours after irradiation, suggesting that the NHEJ pathway, the fast DSB repair pathway [13], might be affected during the process of radioresistance development in MD-PR cells. For verification, reporter cassettes were used to detect the repair efficiency of HR and NHEJ [30].

Firstly, we measured the HR or NHEJ efficiency in MD-PR and MD-PB cells. Results showed that the efficiency of NHEJ pathways was much higher than the efficiency of HR in both cell lines (Fig. 3A, B), which was in accordance with other researchers' results [30]. Secondly, we investigated the efficiency of each DSB repair response pathway in MD-PR and MD-PB cells respectively. No obvious change was observed in HR efficiency in MD-PR cells compared with MD-PB cells (Fig. 3A, B). However, the repair efficiency for NHEJ-I in MD-PB cells was $0.47 \pm 0.06$ and it increased to $0.65 \pm 0.02$ in MD-PR cells, while that for NHEJ-C ascended from $0.62 \pm 0.12$ to $0.91 \pm 0.15$, both with significant difference $(p<0.05)$ (Fig. 3A, B). These results indicated that it is NHEJ, not HR, that is intensely activated in the radioresistant MD-PR cells.
NHEJ is an intricate pathway and many proteins, such as ATM, KU80 and DNA-PKcs, are involved [18, 31]. Expression levels of these key proteins were detected (Fig. 3C) and ATM presented increased level in MD-PR cells. p-ATM (ser1981) is the activated form of ATM, whose existence is scarce without the trigger of DSBs. In both cell lines, the expression level of p-ATM increased dramatically after radiation (Fig. 3C). Consistent with elevated expression of ATM, p-ATM in MD-PR cells was much higher than that in MD-PB cells. No obvious difference in DNA-PKcs and KU80 between these two cell lines was observed (Fig. $3 C)$.

Considering ATM as an important modulator of DDR efficiency and its impact on radiosensitivity [17, 19-21], we next studied the role of incresed expression of ATM in radioresistance development.

\section{ATM mediated the alteration of $\mathrm{Y}-\mathrm{H} 2 \mathrm{AX}$ kinetic and radiosensitivity in MD-PR cells}

To figure out the role of ATM in radioresistance development, we used ATM specific inhibitor to reveal the impact of ATM on elevated radioresistance. CP466722 is an ATM specific inhibitor. The transient application of this compound is proved effective in 
inhibition of cellular ATM kinase activity, resulting in increased cell sensitivity towards radiation [21].

Our experiments showed that CP466722 at the concentration of $8 \mu \mathrm{M}$ was sufficient to inhibit the activation of ATM (Fig. 4A). So $8 \mu \mathrm{M} \mathrm{CP} 466722$ would be used in the following experiments. Plating efficiency was similar with or without CP466722 treatment, indicating this compound had no significant effect on cell plating or cell viability (Fig. S2A). However, the presence of CP466722 did sensitize both MD-PB and MD-PR cells towards radiation, reflected by the surviving fraction. Especially, the inhibition of ATM kinase activity, even transient, re-sensitized MD-PR cells to ionizing radiation to a level similar to that of MD-PB with DMSO (Fig. 4B). Together, these results indicate that increased expression of ATM is correlated with enhanced radioresistance in MD-PR cells.

To evidence the impact of CP422766 on ATM, ATM and p-ATM were tested at the same time (Fig.
4C). No changes were observed for DNA-PKCs since CP466722 is ATM-specific (Data not shown). Since ATM is the main kinase responsible for phosphorylation of $\mathrm{y}-\mathrm{H} 2 \mathrm{AX}$ [32], the application of CP422766 decreased $\mathrm{y}-\mathrm{H} 2 \mathrm{AX}$ level in MD-PR and MD-PB cells after irradiation compared with the control group (Fig. 4C, E, F). Besides, ATM inhibition eliminated $\gamma-\mathrm{H} 2 \mathrm{AX}$ kinetic differences in MD-PR and MD-PB cells (Fig. 4E, F). In both cell lines, $\gamma$-H2AX expression slightly increased and attained a peak at around 4-hour post-irradiation.

Since DSBs repair is mainly conducted by NHEJ in early time [33], We further checked DSB repair efficiency in MD-PB and MD-PR cells with or without CP422766. CP466722 significantly decreased NHEJ-C efficiency in both cell lines, and eliminated the difference of NHEJ-C between two cell lines. (Fig. 4D, Fig. S2B).

\section{B}
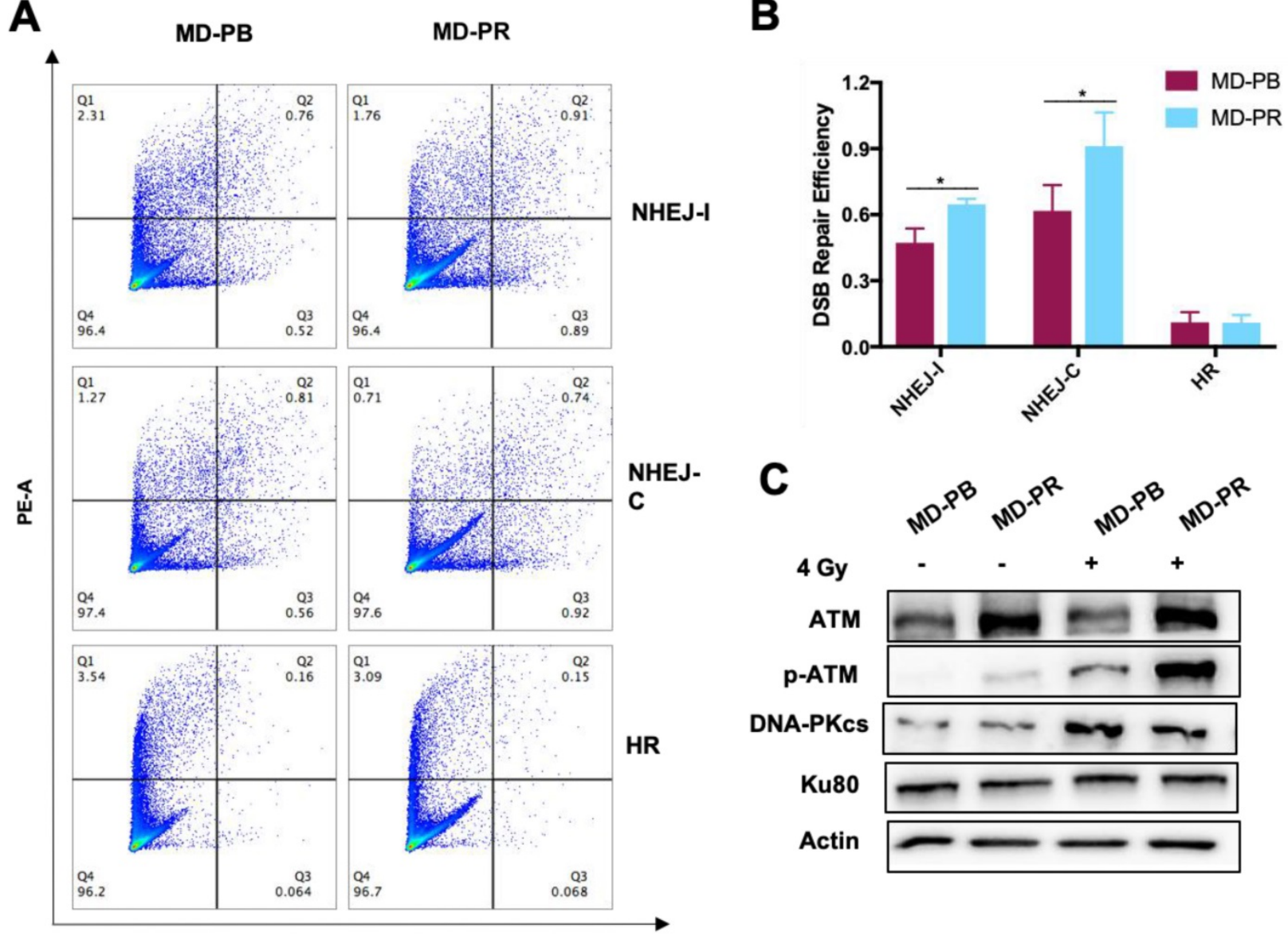

NHEJ-
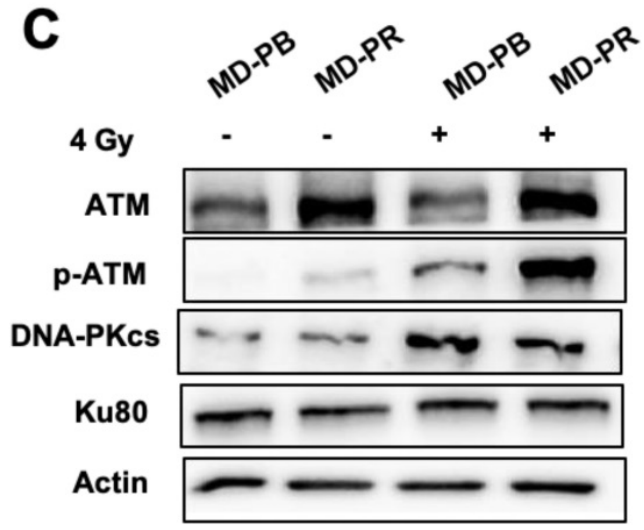

FITC-A

Figure 3. Upregulated DDR efficiency and ATM expression level in MD-PR cells. (A-B) Flow cytometry result of HR and NHEJ efficiency in MD-PR and MD-PB cells. NHEJ with compatible (NHEJ-C) and incompatible (NHEJ-I) ends and HR plasmids were linearized by I-Scel enzyme and transfected into cells with DsRed plasmid as a control. 72 hours later, cells were collected for flow cytometry analysis. The repair efficiency was calculated as cells with green fluorescence (Q2+Q3) / cells with red fluorescence $(\mathrm{Q} 1+\mathrm{Q} 2)$. One representative result of flow cytometry analysis was shown in (A). Statistical results of at least three independent experiments were shown in (B). (C) Western blotting of NHEJ related markers. Cells were subjected to $4 \mathrm{~Gy}$ of radiation or sham-treated. Protein samples were collected for the test. $(* p<0.05)$ 
A

MD-PB

MD-PR

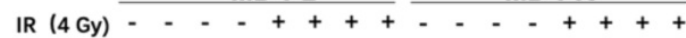

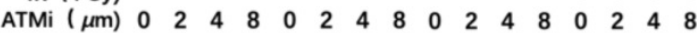
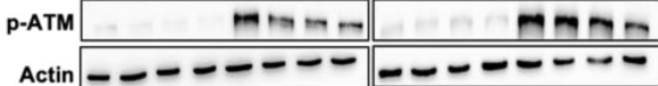

C

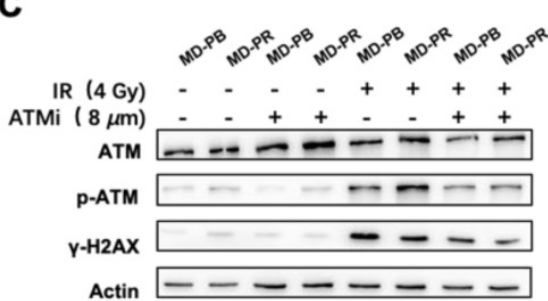

B

E
B

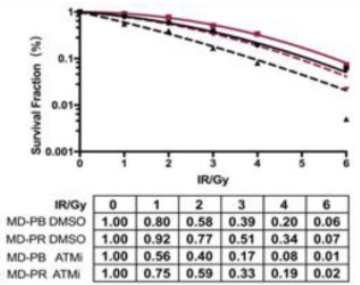

D

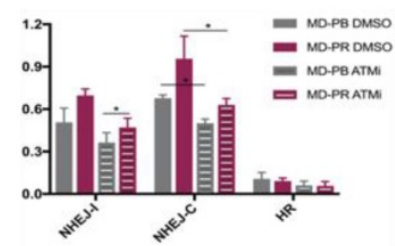

$\mathbf{F}$

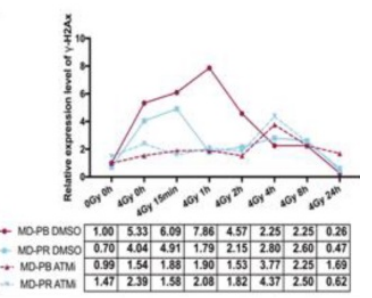

Figure 4. ATM inhibition altered the Y-H2AX kinetic and radiosensitivity in MD-PR cells. (A) Western blotting of p-ATM. Cells were incubated with the indicated concentration of ATMi (CP-466722) for 2 hours, and followed by treatment with or without 4 Gy of X-ray. Protein samples were collected 2 hours after irradiation and subjected to analysis. (B) Representative result of LQM of MD-PR and MD-PB cells with or without ATMi. Cells were incubated with DMSO or $8 \mu M$ ATMi for 2 hours, and followed by exposing to radiation. 3 hours later, medium was completely changed. Surviving fraction was calculated as before and fitted into LQM with statistics shown at the bottom of the panel. (C) Cells were treated as indicated and lysed for western blotting. Samples were collected 1-hour post-irradiation. (D) NHEJ-I, NHEJ-C and HR repair efficiency in cells with DMSO or ATMi. Linearized plasmids and DsRed plasmid were cotransfected. 6 hours later, ATMi or DMSO was added. 72 hours after transfection, flow cytometry analysis was performed. (E) Kinetic of $\mathrm{Y}-\mathrm{H} 2 \mathrm{AX}$ was performed as described elsewhere. Cells were treated with ATMi or DMSO 2 hours before irradiation with the medium completely changed 3 hours post-irradiation. (F) Quantitative analysis of the blots. Plotted is the relative increase of $\mathrm{Y}-\mathrm{H} 2 \mathrm{AX}$ compared with MD-PB at 0 Gy with the treatment of DMSO as a function of post-irradiation incubation time with statistics shown at the bottom.

The high selectivity of CP422766 toward ATM has been documented [21]. Nevertheless, to further confirm the specificity of CP422766, we exploited RNAi-induced knockdown to deplete ATM in MD-PB and MD-PR cells. Si-1 and Si-2 of ATM successfully interfered with the expression of ATM within 72 hours after transfection (Fig. 5A). In keeping with the results of ATM inhibitor, siRNA against ATM increased radiosensitivity in both cell lines and narrowed the gap of radioresistance between MD-PR and MD-PB (Fig. 5B). Apoptosis analysis after lethal irradiation further supported this result (Fig. S2C). The kinetics of $\gamma-\mathrm{H} 2 \mathrm{AX}$ revealed that after ATM knock-down using siRNA, $\mathrm{\gamma}-\mathrm{H} 2 \mathrm{AX}$ was hardly detected in early time after irradiation with a slight peak at around 8-hour (Fig. 5C, D). These results furthur confirmed that altered $\mathrm{\gamma}-\mathrm{H} 2 \mathrm{AX}$ kinetic and radiorensiticity in MD-PR cells were mediated by elevated expression of ATM.

\section{Discussion}

Radiation is one of the most effective therapeutic methods for cancer treatment. However, due to the intrinsic and therapy-induced radioresistance, it remains a conservative choice. In the process of radioresistance development, a pleiotropic of mechanisms are involved [10]. Among them, DDR is one of great importance. Clarifying the mechanism underlying changed DDR in radioresistant cancer cells would provide a new insight into the radiosensitization of cancer cells.

To observe alterations in DDR caused by radiotherapy, we established one radioresistant cell line through repeated $\mathrm{X}$-ray radiation and one cell line as control. These two cell lines have the same origin but present different radiosensitivities (Fig. 1). Significant enhancement in DSB repair efficiency, as reflected by accelerated kinetic of $\gamma-H 2 A X$, was observed in radioresistant cell line MD-PR (Fig. 2A-D). Results from the reporter assays (Fig.3B) suggest that the alteration in $\gamma-\mathrm{H} 2 \mathrm{AX}$ kinetics may be caused by changes in NHEJ efficiency that are responsible for the repair of most DSBs during the early time after irradiation [33]. Analysis of NHEJ related molecules found that ATM expression was increased in MD-PR cells (Fig.3C). Furthur experiments using ATM-specific inhibitor and siRNAs against ATM confirmed the relation between increased ATM expression, altered $\gamma-\mathrm{H} 2 \mathrm{AX}$ kinetic and enhanced radioresistance. Also, MD-PR cells were more resistant to Doxorubicin (Fig. S1F) and Paclitaxel (Fig. S1G), two common chemotherapeutics for breast cancer treatment. Doxorubicin exerts its anti-cancer effect through inducing DNA damage [34]. This further evidenced the enhanced DDR response in MD-PR.

Together, these findings led us to propose that cancers cell may adapt to radiation and develop resistance to irradiation through increasing the expression of ATM, which could accelerate DSBs repair efficiency and reduce radiation-induced cell apoptosis (Fig. 6). Nonetheless, the elevation of ATM just explained part of the reason for enhanced radioresistance. After inhibition of ATM, through specific inhibitor or siRNA, MD-PR cells were still more radioresistant compared with MD-PB cells (Fig. $4 \mathrm{~B}, 5 \mathrm{~B})$, suggesting that some other pathways also contribute to the acquired radio-resistance in MD-PR 
cell line. Of note, compared with ATM inhibitor, the application of siRNAs against ATM further narrowed the gap of radiosensitivity difference between MD-PR and MD-PB. It could be explained by residual ATM kinase activity (Fig. 4A, C) or other functions of ATM independent of its kinase activity [35].

In our experiments, $\gamma-\mathrm{H} 2 \mathrm{AX}$ kinetic was used as the measurement of DSB repair efficiency because higher $\mathrm{y}$-H2AX expression correlates with more unrepaired DSBs [11]. MD-PR cells presented earlier diminishment of $\gamma-\mathrm{H} 2 \mathrm{AX}$ compared with MD-PB, which was in accordance with increased NHEJ repair efficiency. While knock-down of ATM using siRNA abrogated $\gamma-\mathrm{H} 2 \mathrm{AX}$ formation at an early time after irradiation (Fig. 5C), inhibition of ATM with specific inhibitor delayed initial $\gamma-\mathrm{H} 2 \mathrm{AX}$ phosphorylation in both cell lines to around 4 hours post-irradiation (Fig. $4 \mathrm{~F})$. This may be explained by the different treating time between these two experiments. CP466722 was added 2 hours before irradiation and removed 3 hours later, which may cause an increase of $\mathrm{\gamma}-\mathrm{H} 2 \mathrm{AX}$ at 4-hour post-irradiation. siRNAs were transfected 24 hours before cells were reseeded, while the last sample was collected at 72-hour post siRNA transfection, when the effect of the knockdown was still obvious (Fig. 5A). The knockdown of ATM produced detectable residual phosphorylation at the late time point (8-hour, Fig. 5D), which suggested an inhibition of late steps in the DSB process [36].
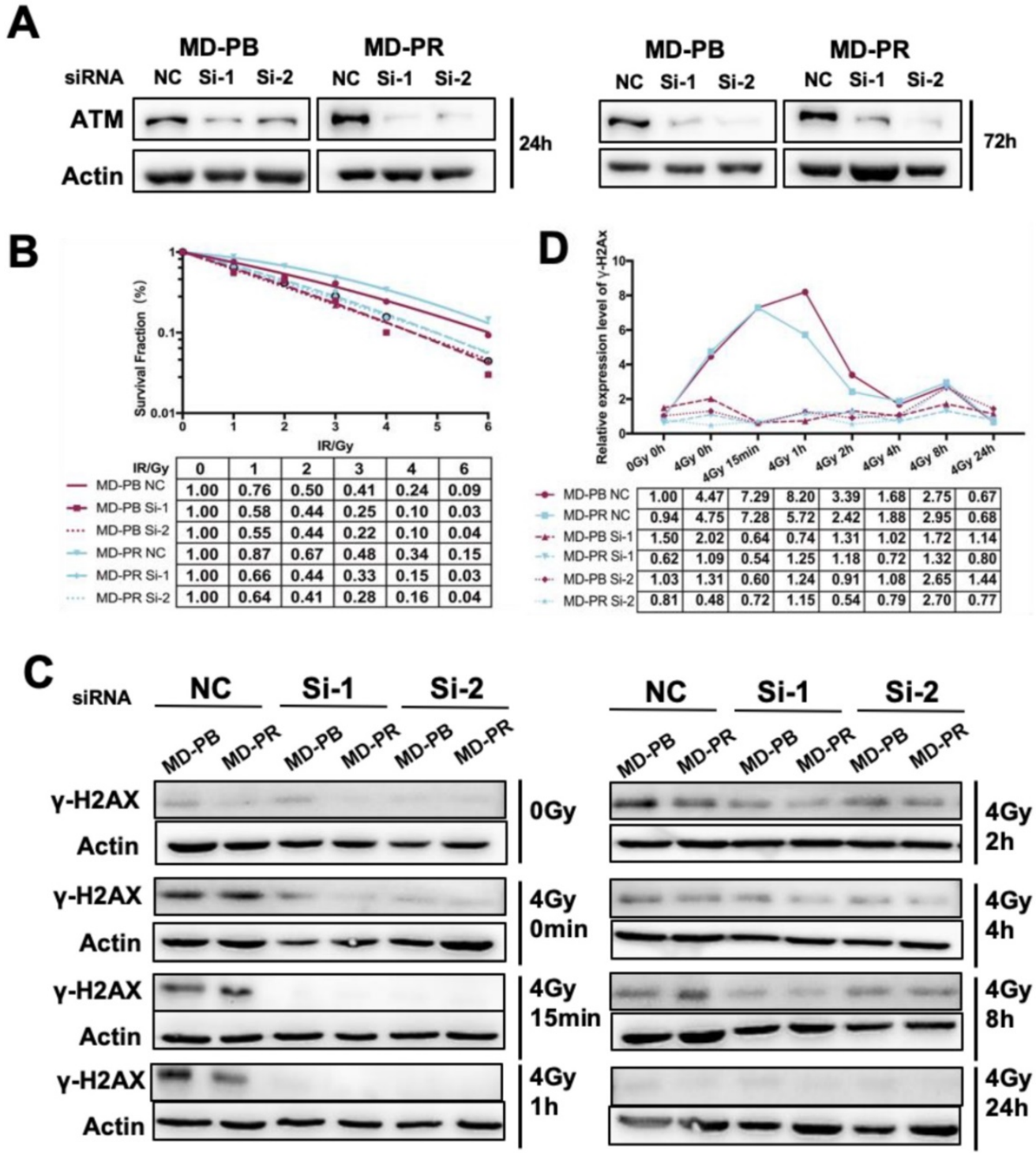

Figure 5. ATM Knock-down sensitized cancer cells to irradiation and abrogated Y-H2AX expression. (A) siRNA against ATM effectively reduced the expression of ATM at up to 72 hours after transfection. Logarithmically growing cells were transfected with siRNA and negative control oligonucleotides. Cells were lysed for ATM analysis at indicated time post-transfection. (B) ATM knock-down sensitized both cell lines to irradiation. Cells were transfected with siRNA or NC as described in Materials and Methods. 24 hours later, cells were seeded in 6-well plate in triplicate and allowed 24-hour for attachment before receiving 0-6 Gy irradiation. Fitting of linear-quadratic model was described in preceding part. (C) ATM knock-down abrogated phosphorylation of H2AX in siRNA treated cells. Cells were transfected with siRNA and reseeded 24 hours later, and allowed another for attachment before subjected to 0 or $4 \mathrm{~Gy}$ of X-ray. Cell lysates were collected at indicated time post-irradiation and used for the analysis of $\mathrm{Y}-\mathrm{H} 2 \mathrm{AX}$. Actin was used as loading control. (D) Quantitative analysis of the blots. Plotted is the relative increase of $\mathrm{Y}-\mathrm{H} 2 \mathrm{AX}$ compared with MD-PB at 0 Gy as a function of post-irradiation incubation time with statistics shown at the bottom. 


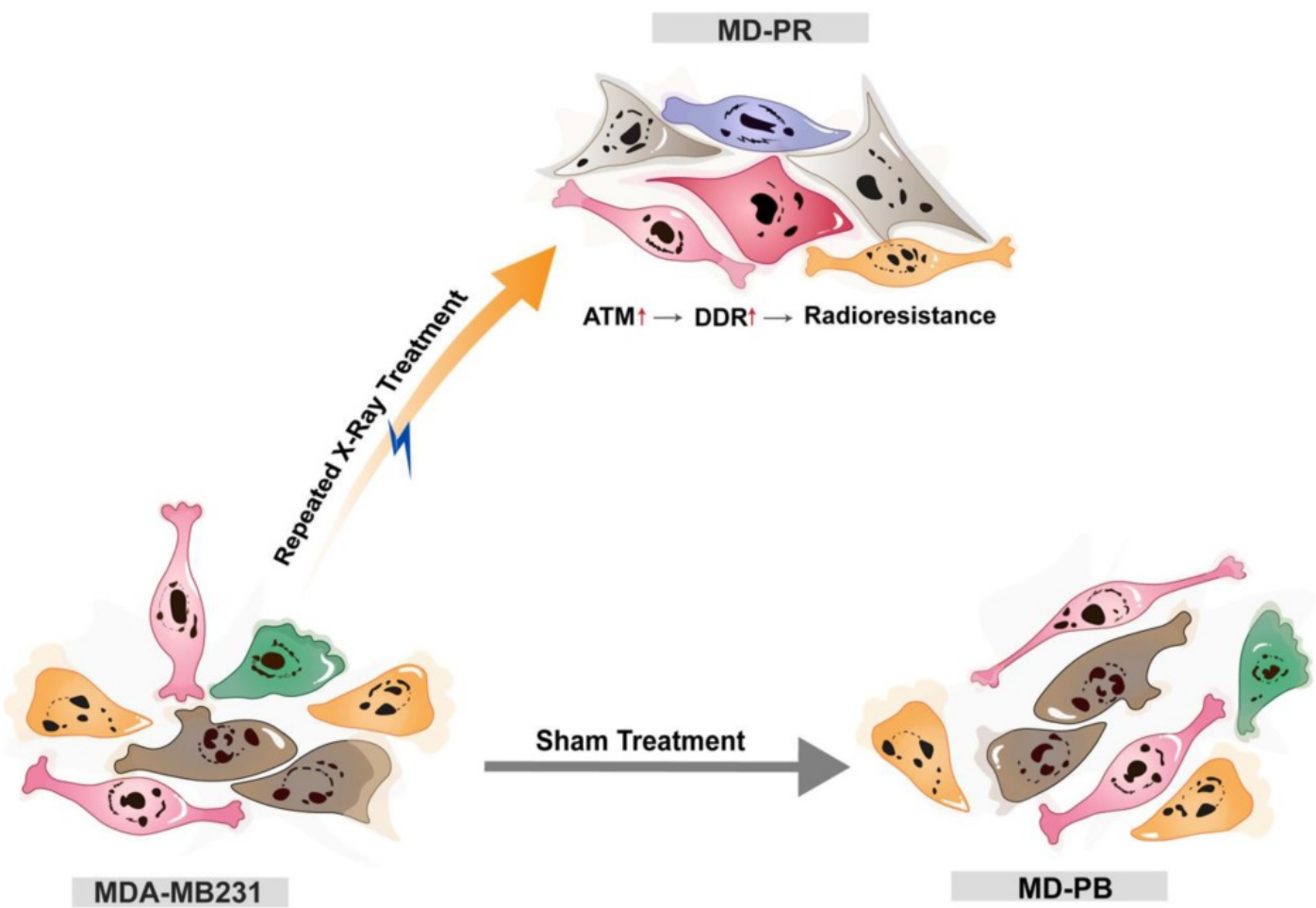

Figure 6. The model of the possible mechanism of radioresistance. Breast cancer cell line MDA-MB231 cells were either sham-treated (MD-PB) or repeated treated with X-ray radiation (MD-PR). In MD-PR cells, the elevation of ATM expression level leads to increased DNA damage repair (DDR) efficiency, which ultimately caused the enhanced radioresistance in MD-PR cells compared with MD-PB cells.

In previous clinical studies, ATM inhibitors have been proved effective in sensitizing tumor cells to radiation and many trials are ongoing [21, 37]. Our findings further support the strategy of targeting ATM alone or in combination with other therapeutics for personized therapy $[38,39]$. ATM is thought to be the core protein for HR [31]. However, elevated expression of ATM did not produce an increased efficiency of HR in our experiments. It is evidenced elsewhere that, ATM is not essential for HR and that HR-driven processes could take place in its absence $[31,40]$. Also, considering HR is strictly restricted to G2 and S phase and the cell cycle distribution had no difference between MD-PB and MD-PR cells, the unchanged HR efficiency is reasonable.

It remains unknown how ATM expression is upregulated in MD-PR cells after repeated radiation treatment. Neither is the mechanism of ATMmediated increased DNA repair efficiency. It is possible that even after a long time since the last radiation treatment, the adaptive effect [41] still exists in MD-PR cells and prepares them for a new even larger dose of radiation and ATM is part of the adaptive response. Future work should focus on ATM conjugated proteins, especially those that can potentially affect DNA damage repair pathways, to better reveal the relation between ATM and increased DDR efficiency in radioresistance development.

\section{Conclusion}

In our present work, we established a relatively radio-resistant breast cancer cell line and proposed an explanation for the emergence of radioresistance in cancer cells and the failure of radiation therapy. When cancer cells receive radiation damage, the repair efficiency of DSBs is upregulated, thus avoiding the fate of apoptosis and necrosis and decreasing radiation sensitivity of cancer. The enhanced repair efficiency might be mediated by elevated expression of ATM and its activated form p-ATM (ser1981). To our knowledge, this is the first evidence correlating elevated DSB repair efficiency with therapy-induced radioresistance.

\section{Abbreviations}

DSBs: double-strand breaks; DDR: DNA damage repair; HR: homologous recombination; NHEJ: non-homologous end joining; ATM: Ataxia telangiectasia mutated.

\section{Supplementary Material}

Supplementary figures and table.

http://www.ijbs.com/v16p1096s1.pdf 


\section{Acknowledgments}

We would like to thank Dr. Vera Gorbunova (University of Rochester, NY) for generously providing NHEJ-I, NHEJ-C and HR constructs.

\section{Funding}

This work was supported by the National Natural Science Foundation of China under Grant 81970094; The Program for Professor of Special Appointment (Eastern Scholar) at Shanghai Institutions of Higher Learning under TP2015022; Shanghai Pujiang Program under Grant 15PJ1404800 and Innovation Program of Shanghai Municipal Education Commission under Grant 15ZZ056.

\section{Author Contributions}

Study design and concept: LD, BL. Data acquisition: BL, MYL, ZMC, GZY. Data analysis and interpretation: BL, MYL, ZMC, ZWW, KX, SYX, WM, YY. Manuscript preparation: BL, LD, WLZ. Manuscript review: BL, LD, YY. All authors read and approved the final manuscript.

\section{Competing Interests}

The authors have declared that no competing interest exists.

\section{References}

1. Bentzen SM. Preventing or reducing late side effects of radiation therapy: radiobiology meets molecular pathology. Nature reviews Cancer. 2006; 6: 702-13.

2. Barnett GC, West CM, Dunning AM, Elliott RM, Coles CE, Pharoah PD, et al. Normal tissue reactions to radiotherapy: towards tailoring treatment dose by genotype. Nature reviews Cancer. 2009; 9: 134-42.

3. Effects of chemotherapy and hormonal therapy for early breast cancer on recurrence and 15-year survival: an overview of the randomised trials. The Lancet. 2005; 365: 1687-717.

4. Effects of radiotherapy and of differences in the extent of surgery for early breast cancer on local recurrence and 15-year survival: an overview of the randomised trials. The Lancet. 2005; 366: 2087-106.

5. Bao S, Wu Q, McLendon RE, Hao Y, Shi Q, Hjelmeland AB, et al. Glioma stem cells promote radioresistance by preferential activation of the DNA damage response. Nature. 2006; 444: 756-60.

6. Roos WP, Kaina B. DNA damage-induced cell death: From specific DNA lesions to the DNA damage response and apoptosis. Cancer letters. 2013; 332: $237-48$.

7. Dahm-Daphi J, Sass C, Alberti W. Comparison of biological effects of DNA damage induced by ionizing radiation and hydrogen peroxide in CHO cells. Int J Radiat Biol. 2000; 76: 67-75.

8. Bohr VA. Deficient DNA repair in the human progeroid disorder, Werner syndrome. Mutat Res. 2005; 577: 252-9.

9. Lavin MF, Shiloh Y. The genetic defect in ataxia-telangiectasia. Annual review of immunology. 1997; 15: 177-202.

10. Tang L, Wei F, Wu Y, He Y, Shi L, Xiong F, et al. Role of metabolism in cancer cell radioresistance and radiosensitization methods. Journal of Experimental \& Clinical Cancer Research. 2018; 37.

11. Bonner WM, Redon CE, Dickey JS, Nakamura AJ, Sedelnikova OA, Solier S, et al. $\gamma \mathrm{H} 2 \mathrm{AX}$ and cancer. Nature Reviews Cancer. 2008; 8: 957-67.

12. Sharma A, Singh K, Almasan A. Histone H2AX phosphorylation: a marker for DNA damage. Methods Mol Biol. 2012; 920: 613-26.

13. Shibata A, Conrad S, Birraux J, Geuting V, Barton O, Ismail A, et al. Factors determining DNA double-strand break repair pathway choice in G2 phase. Embo j. 2011; 30: 1079-92.

14. Branzei D, Foiani M. Regulation of DNA repair throughout the cell cycle. Nature Reviews Molecular Cell Biology. 2008; 9: 297-308.
15. Mao Z, Jiang Y, Liu X, Seluanov A, Gorbunova V. DNA Repair by Homologous Recombination, But Not by Nonhomologous End Joining, Is Elevated in Breast Cancer Cells. Neoplasia. 2009; 11: 683-IN3.

16. Rotman G, Shiloh Y. ATM: A mediator of multiple responses to genotoxic stress. Oncogene. 1999; 18: 6135-44

17. Lavin MF, Kozlov S, Gatei M, Kijas AW. ATM-Dependent Phosphorylation of All Three Members of the MRN Complex: From Sensor to Adaptor. Biomolecules. 2015; 5: 2877-902.

18. Iijima K, Ohara M, Seki R, Tauchi H. Dancing on Damaged Chromatin: Functions of ATM and the RAD50/MRE11/NBS1 Complex in Cellular Responses to DNA Damage. Journal of Radiation Research. 2008; 49: 451-64.

19. Torok JA, Oh P, Castle KD, Reinsvold M, Ma Y, Luo L, et al. Deletion of Atm in Tumor but not Endothelial Cells Improves Radiation Response in a Primary Mouse Model of Lung Adenocarcinoma. Cancer Research. 2019; 79: 773-82.

20. Yan D, Ng WL, Zhang X, Wang $\mathrm{P}$, Zhang Z, Mo Y-Y, et al. Targeting DNA-PKcs and ATM with miR-101 Sensitizes Tumors to Radiation. PLoS ONE. 2010; 5: e11397.

21. Rainey MD, Charlton ME, Stanton RV, Kastan MB. Transient Inhibition of ATM Kinase Is Sufficient to Enhance Cellular Sensitivity to Ionizing Radiation. Cancer Research. 2008; 68: 7466-74

22. Nunez FJ, Mendez FM, Kadilaya P, Alghamri MS, Savelieff M, Koschmann C, et al. IDH1R132H acts as a tumor suppressor in glioma via epigenetic upregulation of the DNA damage response. bioRxiv. 2018.

23. Hennig J, McShane MP, Cordes N, Eke I. APPL proteins modulate DNA repair and radiation survival of pancreatic carcinoma cells by regulating ATM. Cell Death \& Disease. 2014; 5: e1199-e.

24. Enns L, Rasouli-Nia A, Hendzel M, Marples B, Weinfeld M. Association of ATM activation and DNA repair with induced radioresistance after low-dose irradiation. Radiation Protection Dosimetry. 2015; 166: 131-6.

25. Minchom A, Aversa C, Lopez J. Dancing with the DNA damage response: next-generation anti-cancer therapeutic strategies. Therapeutic Advances in Medical Oncology. 2018; 10: 175883591878665.

26. Liu F, Xia Z, Zhang M, Ding J, Feng Y, Wu J, et al. SMARCAD1 Promotes Pancreatic Cancer Cell Growth and Metastasis through Wnt/beta-catenin-Mediated EMT. International journal of biological sciences. 2019; 15: 636-46.

27. Dave B, Mittal V, Tan NM, Chang JC. Epithelial-mesenchymal transition, cancer stem cells and treatment resistance. Breast Cancer Research. 2012; 14.

28. Weichselbaum RR, Ishwaran H, Yoon T, Nuyten DSA, Baker SW, Khodarev $\mathrm{N}$, et al. An interferon-related gene signature for DNA damage resistance is a predictive marker for chemotherapy and radiation for breast cancer. Proceedings of the National Academy of Sciences. 2008; 105: 18490-5.

29. Kim HS, Kim SC, Kim SJ, Park $\mathrm{CH}$, Jeung $\mathrm{H}-\mathrm{C}$, Kim YB, et al. Identification of a radiosensitivity signature using integrative metaanalysis of published microarray data for NCI-60 cancer cells. BMC Genomics. 2012; 13: 348.

30. Mao Z, Bozzella M, Seluanov A, Gorbunova V. Comparison of nonhomologous end joining and homologous recombination in human cells. DNA Repair. 2008; 7: 1765-71.

31. Blackford AN, Jackson SP. ATM, ATR, and DNA-PK: The Trinity at the Heart of the DNA Damage Response. Molecular Cell. 2017; 66: 801-17.

32. Kinner A, Wu W, Staudt C, Iliakis G. Gamma-H2AX in recognition and signaling of DNA double-strand breaks in the context of chromatin. Nucleic Acids Res. 2008; 36: 5678-94.

33. Beucher A, Birraux J, Tchouandong L, Barton O, Shibata A, Conrad S, et al. ATM and Artemis promote homologous recombination of radiation-induced DNA double-strand breaks in G2. The EMBO Journal. 2009; 28 : 3413-27.

34. Thorn CF, Oshiro C, Marsh S, Hernandez-Boussard T, McLeod H, Klein TE, et al. Doxorubicin pathways: pharmacodynamics and adverse effects. Pharmacogenetics and Genomics. 2011; 21: 440-6.

35. Zhang $\mathrm{P}$, Wei $\mathrm{Y}$, Wang L, Debeb BG, Yuan $\mathrm{Y}$, Zhang $\mathrm{J}$, et al. ATM-mediated stabilization of ZEB1 promotes DNA damage response and radioresistance through CHK1. Nature cell biology. 2014; 16: 864-75.

36. Wang $H$, Wang $M$, Wang $H$, Bocker W, Iliakis G. Complex H2AX phosphorylation patterns by multiple kinases including ATM and DNA-PK in human cells exposed to ionizing radiation and treated with kinase inhibitors. Journal of cellular physiology. 2005; 202: 492-502.

37. Hickson I, Zhao Y, Richardson CJ, Green SJ, Martin NMB, Orr AI, et al. Identification and Characterization of a Novel and Specific Inhibitor of the Ataxia-Telangiectasia Mutated Kinase ATM. Cancer Research. 2004; 64: 9152-9.

38. Aguilar-Quesada R, Muñoz-Gámez J, Martín-Oliva D, Peralta A, Valenzuela MT, Matínez-Romero R, et al. Interaction between ATM and 
PARP-1 in response to DNA damage and sensitization of ATM deficient cells through PARP inhibition. BMC Molecular Biology. 2007; 8: 29.

39. Al-Subhi N, Ali R, Abdel-Fatah T, Moseley PM, Chan SYT, Green AR, et al. Targeting ataxia telangiectasia-mutated- and Rad3-related kinase (ATR) in PTEN-deficient breast cancers for personalized therapy. Breast Cancer Research and Treatment. 2018; 169: 277-86.

40. Kass EM, Helgadottir HR, Chen C-C, Barbera M, Wang R, Westermark UK, et al. Double-strand break repair by homologous recombination in primary mouse somatic cells requires BRCA1 but not the ATM kinase. Proceedings of the National Academy of Sciences. 2013; 110: 5564-9.

41. Bonner WM. Low-dose radiation: thresholds, bystander effects, and adaptive responses. Proc Natl Acad Sci U S A. 2003; 100: 4973-5. 